\title{
Multi-Particle Collision Algorithm with Hooke Jeeves applied to the damage identification in a Kabe Problem
}

\author{
Reynier Hernández Torres ${ }^{1}$ \\ Haroldo F. de Campos Velho \\ Instituto Nacional de Pesquisas Espaciais (INPE) São José dos Campos, SP \\ Leonardo D. Chiwiacowsky ${ }^{3}$ \\ Universidade de Caxias do Sul (UCS), Caxias do Sul, RS
}

\begin{abstract}
A new variant of the hybrid metaheutic MPCAHJ (Multi-Particle Collision Algorithm with Hooke-Jeeves method) is presented. Multi-Particle Collision Algorithm is a metaheuristic algorithm that performs a search on the search space. With the addition of the Rotation-Based Learning mechanism to the exploration search, a maior area of the search space has chance to be visited. The Hooke-Jeeves direct search method exploites the best solution found, allowing to achieve better solutions. The performance of all implementation are evaluated over twenty-two well known benchmark functions.
\end{abstract}

Keywords. Hybrid metaheuristic, rotation-based learning, opposition-based learning, multiparticle collision algorithm

\section{Introduction}

Vibration-based damage identification is an application in the field of system identification. In structures and systems, cracks and other damages cause changes in physical properties than can be detectable in the modal parameters (notable frequencies, mode shape, and modal damping).

The damage identification problem can be described as an inverse problem, and solved using optimization techniques. The solution is usually unstable. Small random errors, such as some perturbation on the system or noise in the measurements, can cause large oscillations on the solution.

Metaheuristic algorithms are powerful methods from the Computational Intelligence field that can be applied to many real-world optimization problems.

Multi-Particle Collision Algorithm (MPCA) [1] is a metaheuristic algorithm based on the physics in the nuclear reactor. This algorithm has been successfully used in the solution of optimization problems such as fault diagnosis [2], automatic configuration of neural networks applied to different problems such as atmospheric temperature profile

\footnotetext{
${ }^{1}$ reynier.torres@inpe.br

${ }^{2}$ haroldo@lac.inpe.br

${ }^{3}$ ldchiwiacowsky@ucs.br
} 
identification [3], data assimilation [4], climate prediction [5] and damage identification $[6-8]$.

This work presents the application of the hybrid algorithm Rotation-Based Sampling Multi-Particle Collision Algorithm with Hooke-Jeeves (MPCAHJ), to a bechmark problem in the structure identification area called Kabe Problem.

\section{Hybrid Algorithm: RBSMPCA-HJ}

\subsection{Multi-Particle Collision Algorithm (MPCA)}

MPCA is a optimization algorithm inspired in the physics of the collision inside of a nuclear reactor $[1,9]$. There are to main phenomena occurring: the scattering, where an incident particle could be scattered by a target nucleus, and the absorption, in which the particle could be absorbed by the target nucleus.

MPCA is an populational algorithm, with a set of particles (candidate solutions) travelling inside a nuclear reactor (search space). Each particle is perturbed, creating a new particle that could be absorbed, which means that the previous particle will be substituted by the new, if the new fitness is better. If a perturbed particle is worse than the original one, the particle will be recreated in a new random point within the search space. This process, called as scattering, occurs with a probability depending on the fitness of the particle.

The particles behave cooperatively, strategy called blackboard. The best particle in the set is over-copied for all particles each some number of function evaluations.

A maximum number of function evaluations $\left(N F E_{m p c a}\right)$ is defined as stopping criterion for the MPCA.

The current version MPCA is implemented in FORTRAN 90, and uses MPI libraries for parallel processing.

\subsection{Rotated-Based Learning (RBL) and Rotated-Based Sampling (RBS)}

The RBL concept is an extension of the Opposition-Based Learning (OBL) and the Quasi-Opposition Based Learning (QOBL) mechanisms [10].

The OBL concept was introduced in 2005 by Tizhoosh [11], using the idea that the opposite point of a defined point have a probability of bringing a better solution than the original point in a optimization problem.

After the OBL mechanism, other mechanisms, such as Quase-Opposition Based Learning, Quase-Reflective Based Learning, Center-Based Sampling Learning (CBS), and Rotation-Based Learning have been defined, getting better results than the OBL [10].

All those mechanisms have been applied to improve the performance of some Artificial Intelligence and Computational Intelligence methods, such as Artificial Neural Networks, Fuzzy Logic, and Metaheuristic Algorithms [12].

The Rotation-Based Sampling (RBS) mechanism is a combination of the CBS and RBL mechanisms. 


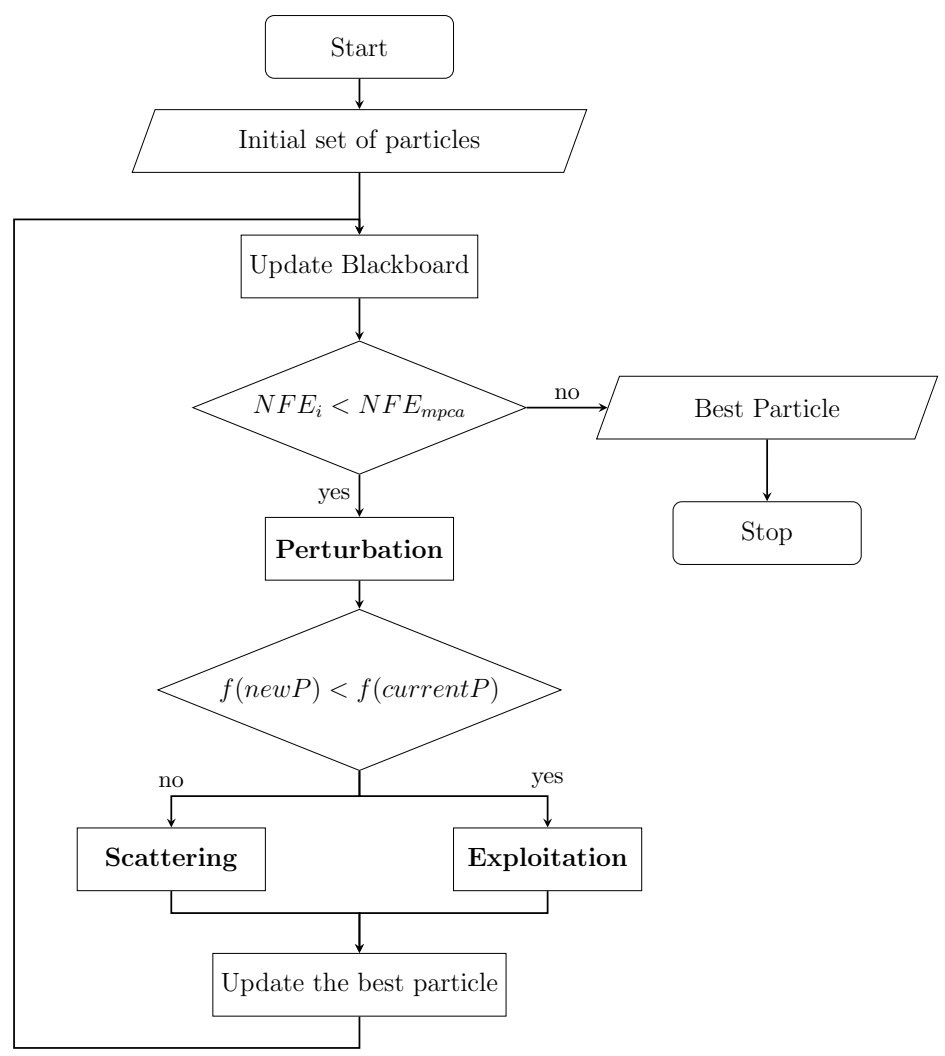

Figure 1: Multi-Particle Collision Algorithm

\section{Hooke-Jeeves Pattern Search Method}

The pattern search method of Hooke-Jeeves (HJ) [13] is a well known algorithm consisting of the repeated application of exploratory moves about a base point which, if successful, is followed by pattern moves. HJ have been used in some hybrid algorithms solving the damage identification problem, such as AS+HJ [14], qG-HJ [7], and MPCA-HJ [6, 8].

Details about the algorithm of HJ can be found in the literature [13].

\section{Empirical Analysis}

\subsection{Experimental configuration}

The experiments were made in a personal computer with $4 \mathrm{x}$ Intel@ Core $^{\mathrm{TM}}$ i7-6500U CPU @ 2.50GHz, with 16 GB of memory, operating with Ubuntu 16.04.2 LTS.

The number of experiments was set in 25 . For the MPCA, the number of particle was set in 10, the blackboard occurs each 100000 function evaluations, the number of function evaluations in the exploitation process is set in 1000, while the $I L$ and $S L$ parameters are set in 0.7 and 1.1, respectively. For the RBS mechanism, $\beta_{0}=3.14 \mathrm{rad}$, and $\delta=0.25$. For the HJ, the parameter $\rho=0.8$, and $h_{\min }=1 \times 10^{-11}$. 


\subsection{Results of the experiments}

The method is tested over a mass-spring system called Kabe Problem. This system include 8 masses and 14 springs in a distribution as shown in Figure 2. Dimensionless values for the masses and stiffnesses are shown in Table 2 .

Experiment were made on noiseless data and data with a $5 \%$ noisy data, both generated synthetically running the direct model. Elements 4 and 7 are simulated as damaged, each one with $10 \%$ of stiffness reduction.

Figure 3 shows the mean of the damages for 25 experiments on the noiseless data. Results are perfect in comparison with the original damage.

Figure 4 shows the boxplot and the Table 1 present the mean and median for the damages for 25 experiments on the noisy data. Both damages were well estimated. For the $5 t h$ and the $6 t h$ elements appeared a dispersion in the estimations, but medians are low for both cases: 0.08 and 1.12, respectively. The mean for the 6 th is affected for three outliers that appeared with values of $50 \%$.

\begin{tabular}{|c|c|c|c|c|c|c|c|c|c|c|c|c|c|c|}
\hline & 1 & 2 & 3 & 4 & 5 & 6 & 7 & 8 & 9 & 10 & 11 & 12 & 13 & 14 \\
\hline Mean & 0.49 & -0.04 & -0.01 & 8.9 & -0.15 & 8.4 & 10.1 & -0.09 & 0.21 & 0.18 & -0.41 & 0.37 & 0.10 & -0.07 \\
\hline Median & -0.01 & 0 & -0.01 & 10 & 0.08 & 1.1 & 10.0 & -0.02 & 0.05 & 0.03 & -0.09 & 0.09 & 0.08 & -0.06 \\
\hline
\end{tabular}

Table 1: Mean and median of the damages estimated for the experiments on the noisy data

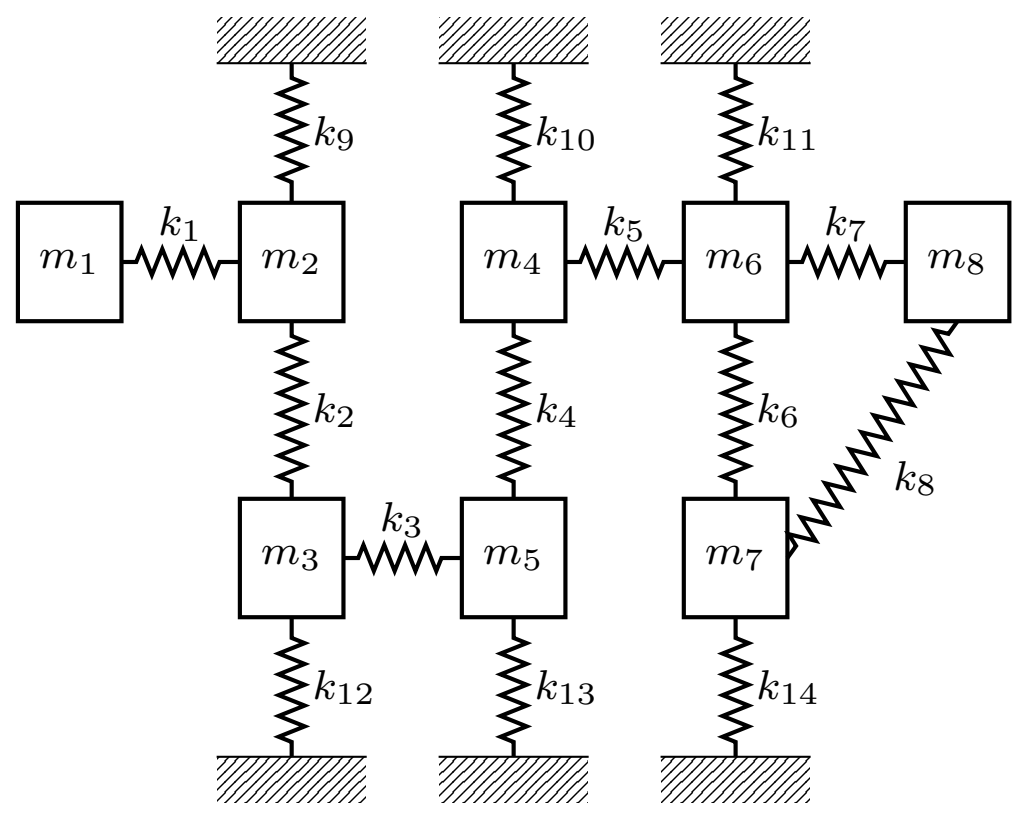

Figure 2: Kabe Problem 


\begin{tabular}{cccccc}
\hline$k_{1,8}$ & $k_{2,6}$ & $k_{3-5}$ & $k_{7}$ & $k_{9,11,12,14}$ & $k_{10,13}$ \\
\hline 1.5 & 10 & 100 & 2 & 1000 & 900 \\
\hline & & & & \\
\cline { 2 - 4 } & $m_{1}$ & $m_{2-7}$ & $m_{8}$ & \\
\cline { 2 - 4 } & 0.001 & 1 & 0.002 & \\
\cline { 2 - 4 } & & &
\end{tabular}

Table 2: Dimensionless mass and stiffness for the Kabe Problem

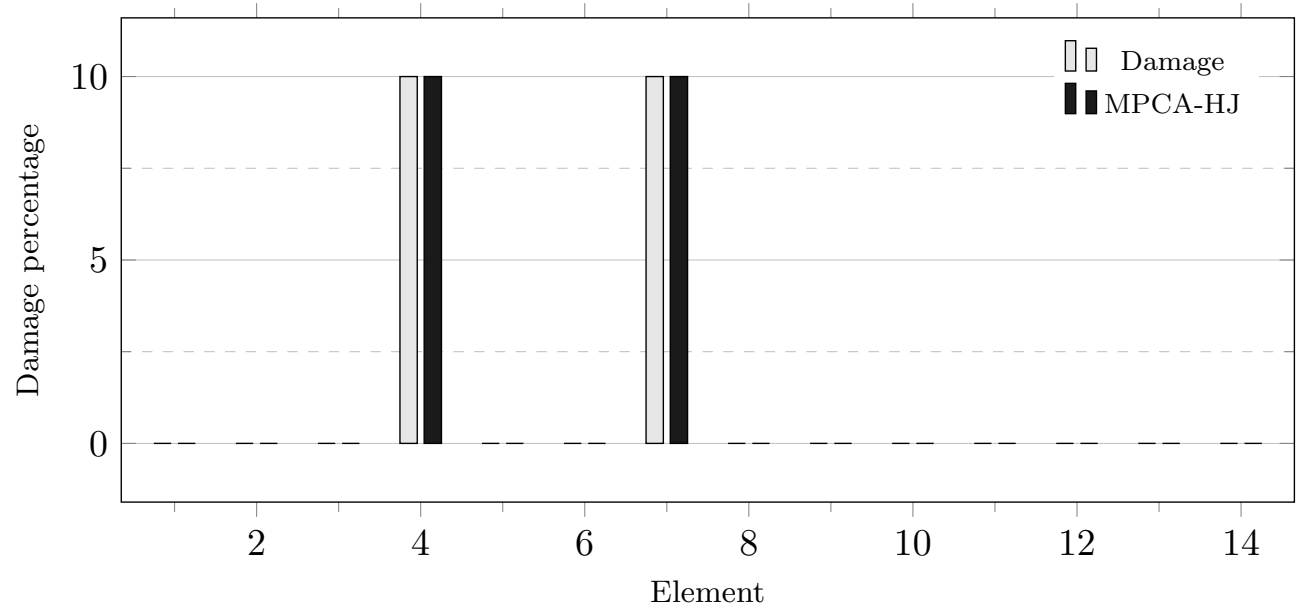

Figure 3: Results for the the Damages identification using RBSMPCA-HJ on the Kabe Problem - Mean for 25 experiments on Noiseless data

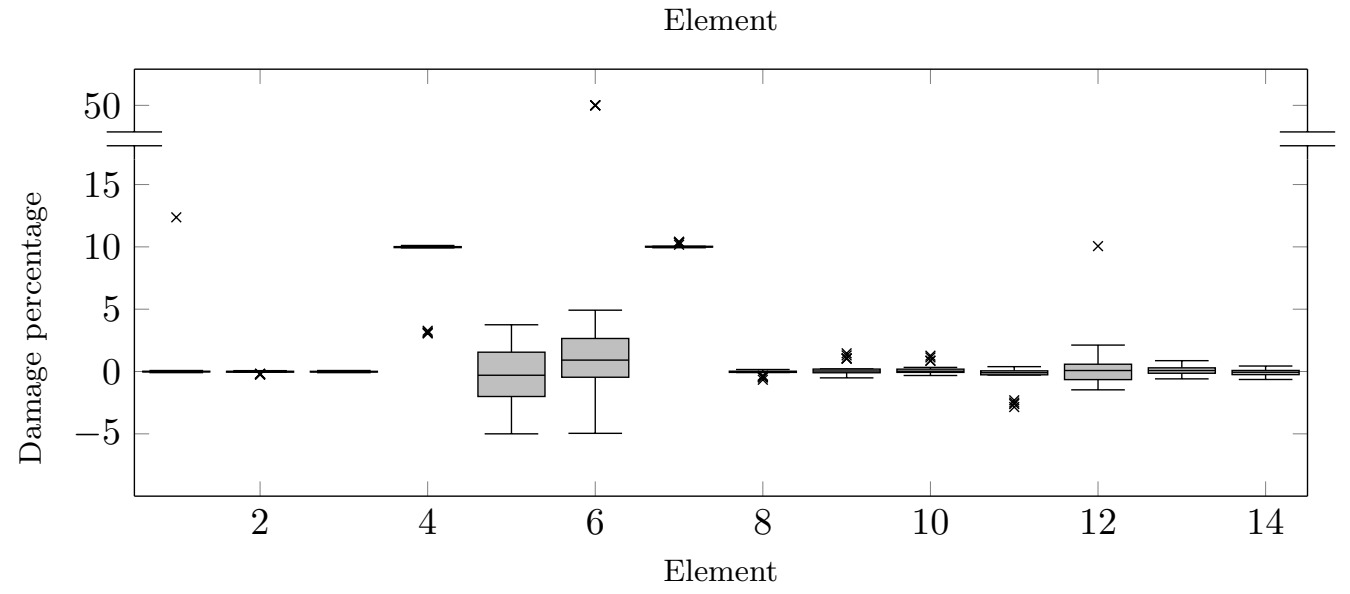

Figure 4: Results for the Damage identification results using RBSMPCA-HJ on the Kabe Problem - Boxplot for 25 experiments on Noisy data 


\section{Final remarks}

In this study, the hybrid metaheuristic RBSMPCA-HJ was applied to the damage identification in the Kabe Problem. Due to the combination of two good methods It takes advantages of the exploration mechanism of the MPCA, with the complement of the Rotation-Based Sampling, and intensification power of the HJ method.

The method was tested over noiseless data and noisy data, obtaining good results in both cases.

\section{Acknowledgements}

The authors acknowledge the financial support provided by the Brazilian institution CNPq, Conselho Nacional de Desenvolvimento Científico e Tecnológico.

\section{References}

[1] E. F. P Luz, J. C. Becceneri, and H. F. Campos Velho. A new multi-particle collision algorithm for optimization in a high performance environment. Journal of Computational Interdisciplinary Sciences, 1(1):3-10, 2008.

[2] L. C. Echevarría, O. Llanes Santiago, and A. J. Silva Neto. Aplicación de los algoritmos Evolución Diferencial y Colisión de Partículas al diagnóstico de fallos en sistemas industriales. Revista Investigación Operacional, 33(2):160-172, 2012.

[3] S. B. M. Sambatti, J. A. Anochi, E. F. P. Luz, A. R. Carvalho, E. H. Shiguemori, and H. F. Campos Velho. Automatic configuration for neural network applied to atmospheric temperature profile identification. In 3rd International Conference on International Conference on Engineering Optimization, pages 1-9, 2012.

[4] J. A. Anochi, H. F. Campos Velho, H. C. M. Furtado, and E. F. P. Luz. Selfconfiguring Two Types of Neural Networks by MPCA. Journal of Mechanics Engineering and Automation, 5:112-120, 2015.

[5] J. A. Anochi and H. F Campos Velho. Optimization of feedforward neural network by Multiple Particle Collision Algorithm. In Foundations of Computational Intelligence (FOCI), 2014 IEEE Symposium on, pages 128-134. IEEE, 2014.

[6] Reynier Hernández, Leonardo D Chiwiacowsky, and Haroldo F Campos Velho. Multiparticle collision algorithm with hooke-jeeves for solving a structural damage detection problem. In A.L. Araúo, J.R. Correia, and C.M. Mota Soares, editors, 10th International Conference on Composite Science and Technology, Lisbon, Portugal, 2015 .

[7] Reynier Hernández, Marluce C Scarabello, Haroldo Campos Velho, Leonardo D Chiwiacowsky, Aline C Soterroni, and Fernando M Ramos. A HYBRID METHOD US- 
ING q-GRADIENT TO IDENTIFY STRUCTURAL DAMAGE. In Ney Augusto Dumont, editor, Proceedings of the XXXVI Iberian Latin-American Congress on Computational Methods in Engineering, Rio de Janeiro, RJ, Brazil, 2015.

[8] Reynier Hernández, Leonardo D Chiwiacowsky, and Haroldo F Campos Velho. Vibration-based Damage Identification using the Multi-Particle Collision Algorithm with Hooke-Jeeves coupled with NASTRAN. In 4th Conference of Computational Interdisciplinary Science (CCIS 2016), São José dos Campos, SP, Brasil, 2015.

[9] W. F. Sacco and C. R. E. Oliveira. A new stochastic optimization algorithm based on a particle collision metaheuristic. Proceedings of 6th WCSMO, 2005.

[10] H. Liu, Z. Wu, H. Li, H. Wang, S. Rahnamayan, and C. Deng. PRICAI 2014: Trends in Artificial Intelligence: 13th Pacific Rim International Conference on Artificial Intelligence, Gold Coast, QLD, Australia, December 1-5, 2014. Proceedings, chapter Rotation-Based Learning: A Novel Extension of Opposition-Based Learning, pages 511-522. Springer International Publishing, Cham, 2014.

[11] H. R. Tizhoosh. Opposition-Based Learning: A New Scheme for Machine Intelligence. In International Conference on Computational Intelligence for Modelling, Control and Automation and International Conference on Intelligent Agents, Web Technologies and Internet Commerce (CIMCA-IAWTIC'06), volume 1, pages 695-701. IEEE, 2005.

[12] Q. Xu, L. Wang, N. Wang, X. Hei, and L. Zhao. A review of opposition-based learning from 2005 to 2012. Engineering Applications of Artificial Intelligence, 29:1-12, 2014.

[13] R. Hooke and T. A. Jeeves. "Direct Search" Solution of Numerical and Statistical Problems. Journal of the ACM (JACM), 8(2):212-229, 1961.

[14] Carlos E. Braun, Leonardo D. Chiwiacowsky, and Arthur T. Gomez. Variations of Ant Colony Optimization for the Solution of the Structural Damage Identification Problem. Procedia Computer Science, 51:875-884, 2015. 